\title{
Hairy cell leukemia variant
}

\section{A description of the spleen morphology and immunophenotype of an archetypical case}

\author{
George Kanellis • Luis Garcia-Alonso • Francisca I. Camacho • \\ Juan Fernando Garcia • Manuela Mollejo • Santiago Montes-Moreno • \\ Jose A. Garcia-Vela • Miguel A. Piris
}

Received: 25 October 2010 / Accepted: 8 November 2010 / Published online: 19 November 2010

(C) Springer-Verlag 2010

\begin{abstract}
Hairy cell leukemia variant (HCLv) is a rare entity ( $10 \%$ of hairy cell leukemia cases) whose diagnostic criteria and appropriate treatment are still uncertain. In this report we aim to add a description of the splenic histology, and extend our knowledge of HCLv. We describe one case of this entity for which a splenic histology was available. It fulfilled all the diagnostic criteria for HCLv, exhibiting a characteristic pattern of splenic infiltration, and responded favorably to treatment. The case described seems to fit well with HCLv where neither morphology nor immunophenotype bears any relation with hairy cell leukemia.
\end{abstract}

Keywords Hairy cell leukemia $\cdot$ Variant $\cdot$ Spleen .

Morphology

Hairy cell leukemia variant (HCLv) is a rare entity first recognized by Cawley et al. in 1980 [1]. It accounts for $10 \%$ of hairy cell leukemia (HCL) cases, with an incidence rate of about 0.03 per 100,000 persons per year [2]. HCLv

\section{G. Kanellis $(\bowtie)$}

Haematopathology Department, Evangelismos Hospital, Perikleus 11,

17343, Athens, Greece

e-mail: gkanellis73@gmail.com

L. Garcia-Alonso • J. A. Garcia-Vela

Department of Haematology, Hospital Universitario de Getafe, Madrid, Spain

F. I. Camacho

Department of Pathology, Hospital Universitario de Getafe,

Madrid, Spain

J. F. Garcia $\cdot$ M. Mollejo • S. Montes-Moreno • M. A. Piris

The Lymphoma Group, Molecular Pathology Program, Centro

Nacional de Investigaciones Oncologicas (CNIO),

Madrid, Spain cases are resistant to conventional HCL therapeutic regimens, such as interferon $\alpha(\mathrm{INF} \alpha)$ and purine analogs, yet have varying responses to other regimens and surgical approaches [3, 4]. HCLv is considered a provisional entity in the new Lymphoma World Health Organization (WHO) Classification [5], under the "splenic lymphoma/leukemia unclassifiable" category, indicating that it awaits more accurate diagnostic criteria to define it fully.

Our patient was admitted and treated at the University Hospital of Getafe, Spain, between 2006 and 2008. The study was done under the supervision of the local Ethical Committee.

A 79-year-old male presented with constitutional symptoms, massive splenomegaly (13 $\mathrm{cm}$ below the costal line), and lymphadenopathy in the cervical and inguinal region. Laboratory studies showed marked leukocytosis $\left(34 \times 10^{9} / 1\right)$ with $25 \times 10^{9} / 1$ total lymphocytes, $1.9 \times 10^{9} / 1$ neutrophils, $1.0 \times 10^{9} / 1$ monocytes, $98 \times 10^{9} / 1$ platelets, $9.6 \mathrm{~g} / \mathrm{dl}$ hemoglobin, $1002 \mathrm{U} / 1 \mathrm{LDH}, 3 \mathrm{mg} / 1 \mathrm{~B} 2$ microglobulin, $32 \mathrm{~g} / 1$ albumin and no $\mathrm{M}$ component. Peripheral blood (PB) cytology consisted of abnormal blastic mononuclear cells each with a round nucleus, abundant basophilic cytoplasm bearing polar prolongations (villous), a conspicuous nucleolus and weak TRAP positivity.

Flow cytometry (FCM) was positive for CD11c (weakly), CD19, CD20, CD22, CD23, CD24, CD79a, FMC7 and lambda, and negative for CD10, CD25, CD38, CD43, CD103 and CD123.

Splenectomy was performed $(2,570 \mathrm{~g}, 33 \times 20 \times 15 \mathrm{~cm})$. Two weeks before the operation the patient received the first of six cycles of R-CHOP. As of December 2008, the patient was in complete remission, with no residual disease, as indicated by the PB smear and FCM.

Bone marrow was easily aspirable, and its morphology showed diffuse infiltration by a mostly large cell population 
with vesicular nuclei and prominent nucleoli. The spleen histology exhibited a relatively monotonous neoplastic proliferation by medium and large cells. The pattern was nodular with diffuse areas mainly in the white pulp that extended into the red pulp with the infiltration of cords and sinuses. Large- and medium-sized cells had a round-to-oval nucleus with no convolutions or contours; the chromatin was dispersed and there was a striking centrally located round eosinophilic nucleolus. The cytoplasm was ample. Immunohistochemical studies on paraffin-embedded spleen sections defined the neoplastic cell population as bcl2(+), CD20(+), DBA44 (wk+), $\operatorname{IgD}(+), \operatorname{IgG}(+)$, Annexin A1(-), bc16(-), CD10(-),CD23(-), CD3(-), CD5(-), Cyclin D1 $(-)$, p53(-) and with low ki-67 (Fig. 1). Cytogenetic analysis showed negativity for BCL2 translocation $\mathrm{t}$ $(14 ; 18)$ and $7 q$ deletion, but indicated a more than $50 \%$ loss of 17p (TP53) in PB.

We have selected this case as archetype of HCL variant, thereby facilitating the recognition of cases with similar features, which may eventually help to define this poorly described large B-cell lymphoma that preferentially involves the splenic red pulp.

The findings involve peripheral blood lymphocytosis, splenomegaly, constitutional syndrome, no autoimmune disorders, an absence of $\mathrm{M}$ component and easily aspirable bone marrow with lymphoid infiltration. No significant cytopenias were detected. On clinical grounds, these features may be considered to be different from those of HCL, and could represent HCLv. However, splenic marginal zone lymphoma (SMZL) and B-cell prolymphocytic leukemia (PLL) are two other entities that should be considered during diagnosis because of their shared attributes.

The morphology fits well with what has been described for HCLv. Thus, PB cell morphology had intermediate features with HCLv and PLL, such as villous projections of the cytoplasm, which are common in HCL and SMZL, and a distinct nucleolus, as is found in PLL. FCM data indicated a mature B cell phenotype, which is more common to HCLv [6], with weak CD11c expression, lambda light chain restriction and negative CD25. TRAP was weakly positive in PB smears, as occurs in $\mathrm{HCL}$ and as reported in some cases of HCLv [2, 7, 8]. The case was positive for $\operatorname{IgG}$ and IgD. Cell morphology differed most strongly from SMZL, where neoplastic cells are smaller, have more condensed chromatin and their cytoplasm, although villous, is scarce and more basophilic. No villous projections have been described in PLL and the cytoplasm is less abundant [3].

A diffuse pattern of infiltration was noted in the bone marrow biopsy, a finding that Matutes et al. [3] have suggested indicates a possible progression.
Fig. 1 a PB smear showing large cells with villous projections and prominent nucleoli. Inset: weak TRAP positivity. b Bone-marrow infiltration, low magnification, diffuse pattern of infiltration, H\&E. c Spleen histology, low magnification indicates nodular pattern of infiltration with diffuse expansion in red pulp, H\&E. d higher magnification, focusing on the red pulp area with obvious sinus and cord infiltration, H\&E. e Even higher magnification shows cell morphology, H\&E. Note the vesicular nucleus, the conspicuous nucleoli and some bizarre cells. f Spleen; CD20-positive neoplastic cells. g Cytoplasmic expression of IgG and IgD (inset) by neoplastic cells. h Low mitotic index (ki-67)

The spleen morphology showed a background of infiltration of the red pulp, with extensive infiltration of the red pulp sinuses and splenic cords, and effacement of the white pulp by the neoplasm, which nevertheless appeared to display some conspicuous micronodules corresponding to preexistent follicles. Cytology revealed a population of medium-to-large cells with prominent nucleoli and abundant cytoplasm. In contrast, SMZL has a distinct biphasic morphology occupying the white pulp with some extension to the red pulp. Cytologically, it is composed of two populations of cells, neither of which resembles those described in our case [9]. Prolymphocytic leukemia is characterized by the presence of massive splenomegaly, absence of lymphadenopathy, marked lymphocytosis (usually $>100 \times 10^{9} / 1$ ), anemia and thrombocytopenia, with circulating prolymphocytes exceeding $55 \%$ of the lymphoid cells [10], and splenic histology showing diffuse infiltration by prolymphocytes.

Response to therapy and follow-up showed a remarkably good response to the treatment of choice for HCLv, which was a combination of splenectomy and R-CHOP [2, 11], with complete remission from the disease at the time of writing. His laboratory profile, PB and tissue morphology, immunohistochemistry and clinical outcome are closely consistent with those of HCLv.

In conclusion, we selected a potential case with which to review and discuss the problems of the recognition and differential diagnosis of HCLv. Our case seems to fit well with HCLv and is consistent with previously published data, thus adding a description of the splenic histology, and extends our knowledge of this entity, whereby neither morphology, immunophenotype nor response to therapy appear to be related to HCL. Substantial differences with HCL likely recommend to consider a different, more appropriate name, for this entity. Some cases with these features could have been denominated in the past as PLL. A reevaluation of the limits between HCLv and PLL is warranted, after taken into account the biological heterogeneity of PLL [10], and the exclusion of PLL cases with $t$ $(11 ; 14)(\mathrm{q} 13 ; \mathrm{q} 32)$ translocation, now considered as blastic variants of MCL [10]. 

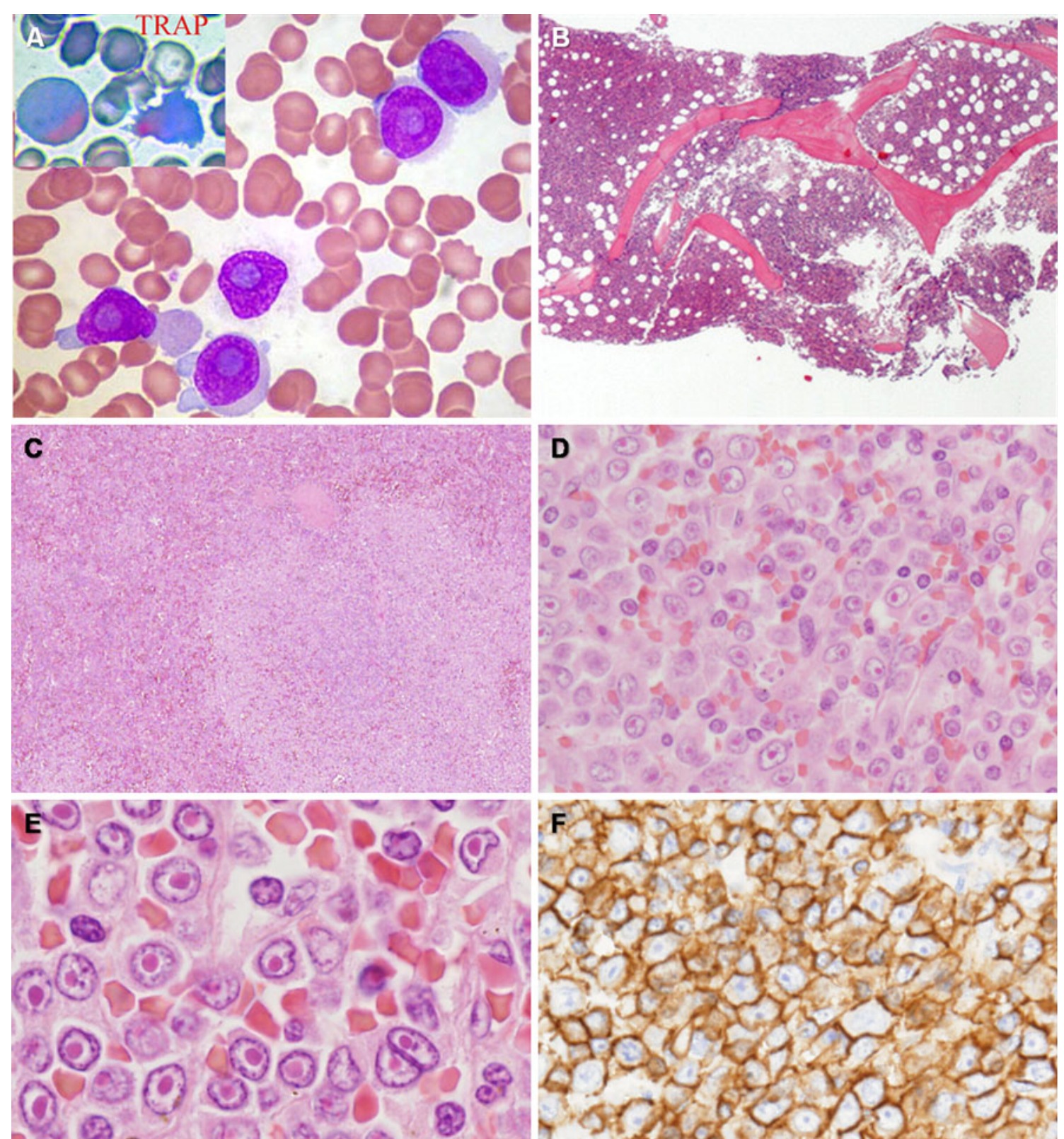

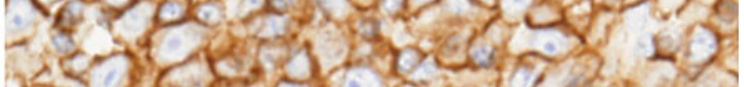

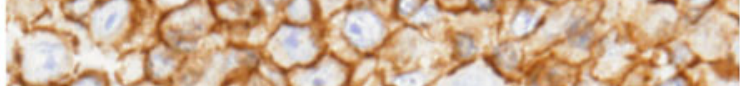

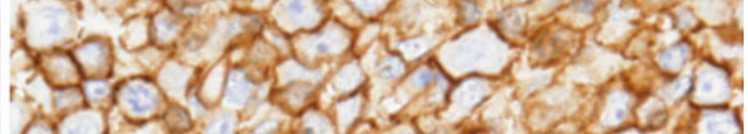

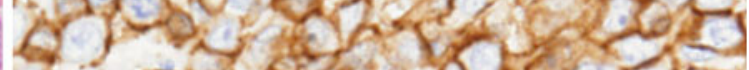

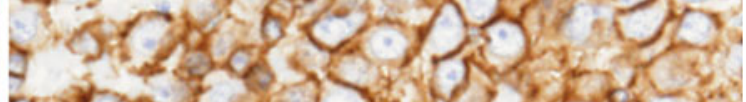

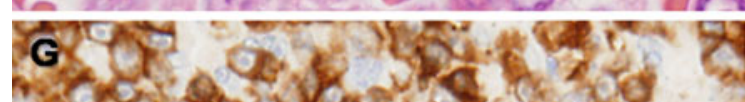

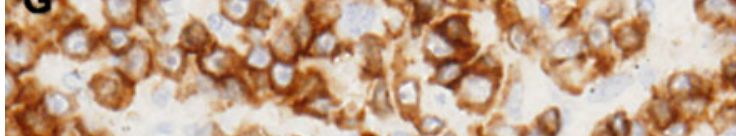

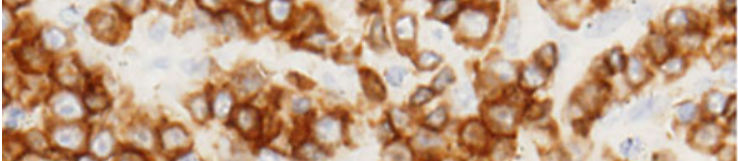

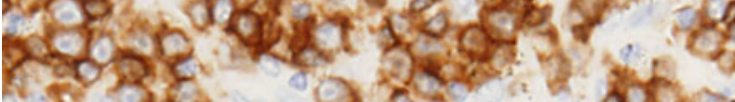
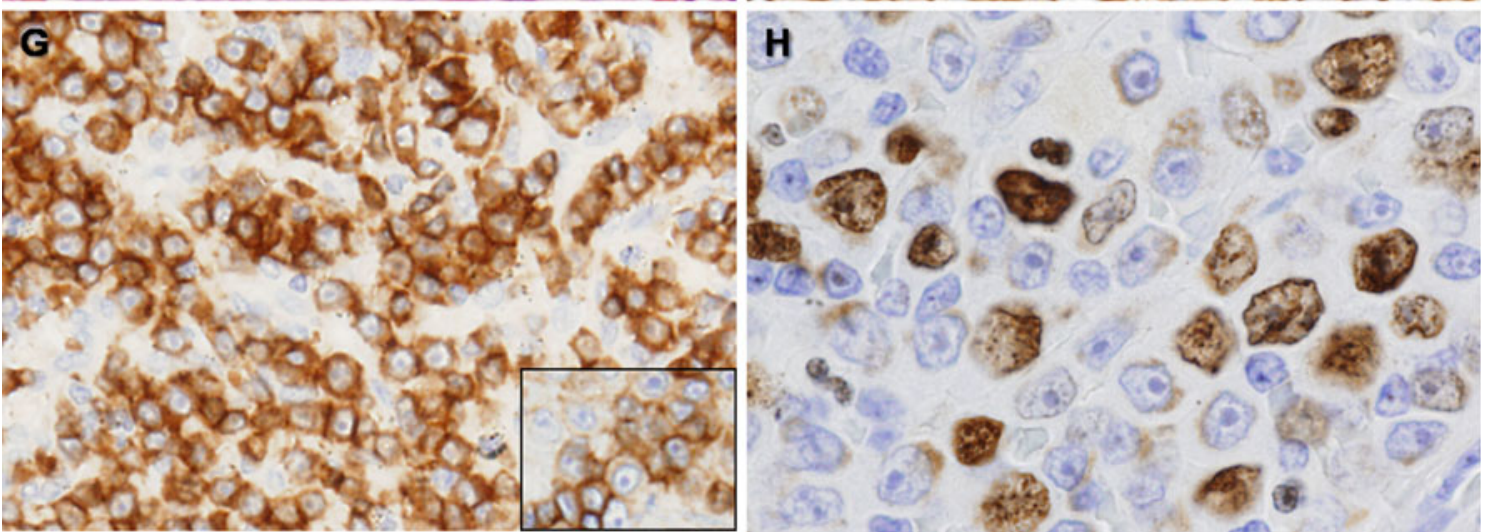
Acknowledgements This study was supported by the Instituto de Salud Carlos III, Ministerio de Sanidad y Consumo (RETICS, PI051890, PI051623, PI052800), and the Ministerio de Ciencia y Tecnología (SAF2005-00221, SAF2008-03871), Spain.

Conflict of interest The authors declare that they have no conflict of interest.

\section{References}

1. Cawley JC, Burns GF, Hayhoe FG (1980) A chronic lymphoproliferative disorder with distinctive features: a distinct variant of hairy-cell leukaemia. Leuk Res 4(6):547-559

2. Robak T (2006) Current treatment options in hairy cell leukemia and hairy cell leukemia variant. Cancer Treat Rev 32(5):365-376

3. Matutes E, Wotherspoon A, Brito-Babapulle V, Catovsky D (2001) The natural history and clinico-pathological features of the variant form of hairy cell leukemia. Leukemia 15(1):184-186

4. Sainati L, Matutes E, Mulligan S, de Oliveira MP, Rani S, Lampert IA et al (1990) A variant form of hairy cell leukemia resistant to alpha-interferon: clinical and phenotypic characteristics of 17 patients. Blood 76(1):157-162
5. Piris MA, Foucar KM, Mollejo M, Campo E, Falini B (2008) Splenic lymphoma/leukemia, unclassifiable. In: Swerdlow SH, Campo E, Harris NL, Jaffe ES, Pileri SA, Stein H et al (eds) WHO classification of Tumours of Haematopoietic and Lymphoid Tissues. IARC Press, Lyon

6. Cessna MH, Hartung L, Tripp S, Perkins SL, Bahler DW (2005) Hairy cell leukemia variant: fact or fiction. Am J Clin Pathol 123 (1):132-138

7. Matutes E, Wotherspoon A, Catovsky D (2003) The variant form of hairy-cell leukaemia. Best Pract Res 16(1):41-56

8. Del Giudice I, Matutes E, Morilla R, Morilla A, Owusu-Ankomah $\mathrm{K}$, Rafiq $\mathrm{F}$ et al (2004) The diagnostic value of CD123 in B-cell disorders with hairy or villous lymphocytes. Haematologica 89 (3):303-308

9. Matutes E, Oscier D, Montalban C, Berger F, Callet-Bauchu E, Dogan A et al (2008) Splenic marginal zone lymphoma proposals for a revision of diagnostic, staging and therapeutic criteria. Leukemia 22(3):487-495

10. Del Giudice I, Davis Z, Matutes E, Osuji N, Parry-Jones N, Morilla A et al (2006) IgVH genes mutation and usage, ZAP-70 and CD38 expression provide new insights on B-cell prolymphocytic leukemia (B-PLL). Leukemia 20(7):1231-1237

11. Narat S, Gandla J, Dogan A, Mehta A (2005) Successful treatment of hairy cell leukemia variant with rituximab. Leuk Lymphoma 46 (8):1229-1232 